\title{
Evaluation of dosimetric implications of Pareto and constrained mode of optimization for Monaco TPS generated VMAT plans in post operated carcinoma of the left breast
}

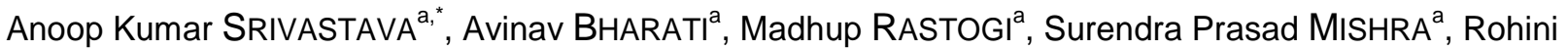 \\ KHURANA $^{\mathrm{a}}$, Rahat HADI ${ }^{\mathrm{a}}$, Ajeet Kumar GANDHI ${ }^{\mathrm{a}}$, Lalatendu MISHRA ${ }^{\mathrm{a}}$ \\ ${ }^{a}$ Department of Radiation Oncology, Dr. Ram Manohar Lohia Institute of Medical Sciences, Lucknow India-226010 \\ *E-mail address: dranooprmlims@gmail.com
}

\begin{abstract}
Intensity-modulated radiotherapy (IMRT) is being practiced for the last several years with a special approach for radiation therapy in post-mastectomy breast cancer patients. Meeting the cardiac dose constraints has always been a challenge during radiotherapy planning by both IMRT and VMAT (volumetric modulated arc therapy) of postmastectomy left breast patients. With the advancement in IMRT planning techniques, it has been modified to VMAT with more degrees of freedom for modulation and is being utilised more frequently. This helps in obtaining a suitable plan for achieving both the dose homogeneity in target volume and dose constraints to Organ at Risk (OAR). 10 Patients with carcinoma of the left breast (post-mastectomy) were selected for this study. VMAT treatment plans for these patients were generated for $6 \mathrm{MV}$ photons on the Monaco treatment planning system (TPS) using two types of optimization modes i.e. Pareto and Constrained mode available in Monaco TPS. For comparative dosimetric evaluation of the efficacy of these two types of optimization modes similar calculation algorithms, calculation grids, arcs, and beam sequencing parameters were used for generating treatment plans. The dosimetric quantities such as volume receiving more than $95 \%$ of the prescribed dose $\left(\mathrm{V}_{95}\right)$, volume receiving more than $107 \%$ of the prescribed dose $\left(\mathrm{V}_{107}\right)$ and Maximum dose $\left(\mathrm{D}_{\max }\right)$ for target volume, mean dose $\left(\mathrm{D}_{\text {mean }}\right)$ for heart, volume receiving more than $30 \mathrm{~Gy}\left(\mathrm{~V}_{30}\right)$ volume receiving more than $20 \mathrm{~Gy}\left(\mathrm{~V}_{20}\right)$ volume receiving more than $5 \mathrm{~Gy}\left(\mathrm{~V}_{5}\right)$ for ipsilateral lung and total monitor units delivered were analysed for both optimization modes. A judicious mix of multiple planning parameters and variables using these two modes of optimization was applied and recorded. Both optimization modes yielded similar outcomes. However, Pareto mode has shown better coverage for planning target volume (PTV) with comparable doses to OARs.
\end{abstract}

Key words: VMAT; optimization; constrained; Pareto; Monte-Carlo.

\section{Introduction}

Intensity Modulated Radiotherapy (IMRT) has been in practice for more than the past two decades with specialised approach for the treatment of post-mastectomy breast cancer. The topology of post-mastectomy breast patients is complex and achieving both the dose homogeneity to the target volume and dose constraints to OARs pose great challenges in treatment planning. Meeting the dose constraints for the heart has always been a challenge for radiotherapy planning of post-mastectomy left breast patients for both IMRT and VMAT. As planning and delivery techniques progress VMAT proved to be more suitable for creating an optimal treatment plan in this location and has become a more often utilized technique. Multi-arc VMAT is used more often as it can provide plans with better dose homogeneity within the target and helps in achieving dose constraints to Organ at Risks (OAR). VMAT provides more degree of freedom, thanks to changes in the speed of gantry rotation as well as changes in dose rates. The multiple degrees of freedom in modulation enables the preparation of treatment plans with a reduced number of monitor units compared to standard IMRT. Jonathan Bogue et $\mathrm{al}^{1}$ in their study compared the dosimetric data of VMAT with integrated skin flash to 3D field-in-field tangents for left breast irradiation and advocated that VMAT may be more selectively applied for left breast irradiation. They even reported that it may even be equally effective without respiratory gating. VMAT spares the heart in a better manner and meet dose constraints to OARs more effectively while facilitating the delivery of prescription dose in the target volume even in cases of difficult anatomical geometry. Vishruta A. Dumane et $\mathrm{al}^{2}$ reported that a combination of VMAT and Deep Inspiration Breath Hold (DIBH) had significant dosimetric gains and results in 
diminishing the dose to the heart, lungs and contralateral breast/implant. They postulated that VMAT with DIBH may also be beneficial in breast cancer patients with implant reconstructions. Mikel Byrne et $\mathrm{al}^{3}$ illustrated that semiautomated tangential VMAT is a useful addition to the treatment options available for breast/chest wall and nodal irradiations compared to the 3 Dimensional Conformal Radiation Therapy (3DCRT). Shyama Prem Sudha et $\mathrm{al}^{4}$ observed that VMAT is dosimetrically superior to the field in field 3DCRT for left breast cancer patients. This facilitates comparable PTV coverage and better sparing of the heart and lung. Antonella Fogliata et $\mathrm{al}^{5}$ in their study discussed the various dosimetric trade-offs in breast treatment with the VMAT technique. Although there are various schools of thought regarding radiotherapy of left-sided post-mastectomy breast cancer, there is no doubt that VMAT is now a wellestablished modality of treatment in selected cases. Mac Clements et $\mathrm{al}^{6}$ in a dosimetric study explained various modes of optimization available in the Monaco treatment planning system namely Constrained and Pareto optimizations. In constrained mode OAR constraints are achieved first then target coverage. Whereas in Pareto mode, Monaco prioritizes target coverage, and then the system seeks to meet the constraints in the OAR. David Craft et $\mathrm{al}^{7-9}$ in their studies deliberated a technique known as Pareto surface navigation, which allows physicians and treatment planners to get the best balance between target coverage and minimization of doses to OAR. They also describe two other methods i.e. goal programming and prioritized optimization. Two other methods are designed to handle multiple conflicting objectives. They also considered the Pareto surface approach for multi-criteria optimization (MCO) intensity-modulated radiation therapy (IMRT) treatment planning, where a database of Pareto optimal plans is generated for the patient and then that database is interactively navigated by the treatment planner or physician. The IMRT plans resulting from the use of biological cost functions were compared with plans obtained with dosevolume based optimization. This study aims to compare the dosimetric quantities of different modes of optimization i.e. Pareto and constrained available in Monaco Treatment planning system (TPS) for VMAT of left side post-mastectomy breast patients.

\section{Materials and Methods}

A group of 10 patients with left breast cancer (post operatedmodified radical mastectomy) were selected for this study. The patients were simulated in treatment position using appropriate immobilization on CT simulator (Somatom sensation Open 16 Slice CT, Siemens Healthinears, Germany). Slices of $3 \mathrm{~mm}$ thickness were obtained inferiorly from $3 \mathrm{~cm}$ below the xiphisternum to hyoid bone superiorly. Subsequently, simulation plan data were transferred to Monaco TPS. The radiotherapy plans were generated in Monaco IMRT Optimizer 5.11.02 (6 MV beam library of linac Elekta infinity (Elekta Medical Systems, Crawly, UK make) using both optimisation methods.

\section{Monaco treatment planning system ( $\mathrm{v}$ 5.11.02.)}

Monaco is one of the first commercially available and clinically applied IMRT treatment planning systems which enables biological optimization. In addition to physical cost functions commonly used in IMRT TPS, such as dose-volume histogram (DVH) constraints and maximum dose, Monaco offers three biological cost functions for obtaining the optimised plan.

The physical cost function based dose optimization is based on several statistics of dose distribution (eg. maximum dose, minimum dose, mean dose). Biological optimization is assumed to be based on the maximization of the tumour control probability (TCP) and minimization of the normal tissue complication probability (NTCP). In fact, often surrogates for these values, for example the Equivalent Uniform Dose (EUD), are used instead of TCP and NTCP. The biological cost functions employed in Monaco are Poisson and use the statistics like cell kill model, the serial complication model, and the parallel complication model. Summary of cost functions used to design the Monaco plans are listed in Table 1.

Table 1. Summary of cost functions used in Monaco

\begin{tabular}{|c|c|c|c|}
\hline Applicability & Model Name & Parameters and iso-constraints & Description \\
\hline \multicolumn{4}{|c|}{ Biological Cost functions } \\
\hline Target & Poisson Cell kill model & $\begin{array}{l}\text { Cell sensitivity }(0.1-1.0) \\
\text { EUD prescription (Gy or cGy) }\end{array}$ & $\begin{array}{l}\text { Mandatory cost function for targets; } \\
\text { no penalty for hot spot }\end{array}$ \\
\hline OARs & Serial complication model & $\begin{array}{l}\text { Power law exponent }(>1) \\
\text { Equivalent uniform dose (Gy or cGy) }\end{array}$ & Penalizes for hot spot \\
\hline OARs & Parallel complication model & $\begin{array}{c}\text { Reference dose (Gy or cGy) Power law exponent }(>1) \\
\text { Mean organ damage }(\%)\end{array}$ & Effective for reducing mean organ dose \\
\hline \multicolumn{4}{|c|}{\begin{tabular}{|l} 
Physical Cost Function \\
\end{tabular}} \\
\hline Target or OARs & Quadratic overdose penalty & $\begin{array}{l}\text { Maximum dose (Gy or cGy) } \\
\text { Root mean square excess dose (Gy or cGy) }\end{array}$ & Penalizes for hot spot with some leniency \\
\hline Target or OARs & Maximum dose & Maximum dose (Gy or cGy) & Penalizes for hot spot with zero leniency \\
\hline OARs & Overdose Volume Constraints & $\begin{array}{l}\text { Threshold dose (Gy or cGy) } \\
\text { Volume }(\%)>\text { Threshold dose }\end{array}$ & $\begin{array}{l}\text { Forces a DVH through } \\
\text { or below a single point }\end{array}$ \\
\hline
\end{tabular}


For biologically optimal dose distribution Monaco employs a two-stage process for the optimization of dose distributions. In the first step, the fluence distribution of IMRT beams is optimized. In the second step, the beam segmentation is optimized. This also employs a finite size pencil beam algorithm for beamlet fluence optimization and may also be used for fast calculation of dose distribution from segmented beams. In the second step, for more accurate dose calculations, Monaco offers a Monte Carlo dose calculation engine based on the XVMC code19 which utilises virtual energy fluence (VEF) model of the treatment head of the accelerator.

\section{Treatment Planning}

All the patients in this study were planned using the Monaco TPS. The target volume and organ at risk (OAR) were delineated slice by slice on the contouring workstation. The OARs delineated were heart and lung according to RTOG protocol. ${ }^{10}$ Before running the optimization the treatment unit, beam energy, plan algorithm, grid size, number of arcs, arcs directions (clockwise or anticlockwise), and arc length were defined. The sequencing parameters available in planning options are enabled to control the maximum number of arc, maximum number of control points of each arc, minimum segment width, and degree of fluence smoothing. Thus, in this study for evaluations of the efficacy of both types of optimizations, we used the same parameters of calculations and sequencing and similar IMRT parameters. Treatment plans were generated with two arcs for x-ray photon beam of $6 \mathrm{MV}$ energy of Elekta Infinity ${ }^{\mathrm{TM}}$ linear accelerator (M/s Elekta Medical Systems, Crawly UK). This linear accelerator is equipped with 40 pairs of multileaf collimator having 1.0 centimetre leaf width at isocenter. The dose of $50 \mathrm{~Gy}$ in 25 fractions over a period of 5 weeks was prescribed to PTV. Cost functions for target dose optimizations were described in terms of the equivalent uniform dose (EUD) and quadratic overdose. For all patients, two-step optimization was utilised with a dose calculation grid of $0.3 \mathrm{~cm}$. In MONACO TPS the default normalization of the dose is the normalization to isocenter but as we all are well aware that in the case of VMAT this prescription is the only deciding factor for a number of fractions to be treated. The actual prescription is in terms of target EUD in the IMRT constraints tab. Acceptance criteria chosen for both the plans were $95 \%$ of PTV to be covered by $95 \%$ of the prescribed dose. Two VMAT plans, one with a constrained mode of optimization, and another with Pareto mode of optimization were generated. All the dosimetric analysis were on the basis of the International Commission of Radiological Units and Measurements (ICRU) 50 and 62. The specific cost function, maximum dose along with optimized over all voxels option was applied to the patient body contour to minimize high dose volume both in the target and patient body.

\section{Plan evaluation and statistics}

Treatment plans obtained for both methods of optimization for every patient was evaluated by comparison of several dosimetric parameters in the target and the Organs at Risk. Optimal plans generated with Pareto and Constrained modes were analyzed in terms of different dosimetric quantities for the target as well as OARs. The dosimetric quantities were obtained for $\mathrm{V}_{95}, \mathrm{~V}_{107}$ and $\mathrm{D}_{\max }$ for target volume, $\mathrm{D}_{\text {mean }}$ for heart, $\mathrm{V}_{30}, \mathrm{~V}_{20}$ and $\mathrm{V}_{5}$ and total monitor units. To compare results obtained with Pareto and constrained mode of optimization non-parametric Wilcoxon signed-rank test was used (SPSS software).

\section{Results}

The results were analyzed and its statistical outcomes were evaluated. Graphical presentation of various planning parameters was plotted for easy assimilations of dosimetric analysis and are presented in Figures 1-8. The summary of the results is presented in Table 2.

Table 2. $p$ value in order from most significant to least significant

\begin{tabular}{|c|c|c|c|}
\hline \multirow{2}{*}{ Parameters } & \multicolumn{2}{|c|}{ Mean \pm SD } & \multirow{2}{*}{ p Value } \\
\hline & Constrained & Pareto & \\
\hline $\mathrm{V}_{95}$ for PTV & $94.6 \pm 2.1$ & $96.6 \pm 1.6$ & 0.007 \\
\hline $\mathrm{V}_{20}$ for lung & $35.6 \pm 5.2$ & $40.4 \pm 3.8$ & 0.065 \\
\hline $\mathrm{V}_{5}$ for lung & $66.0 \pm 6.0$ & $70.1 \pm 4.5$ & 0.083 \\
\hline $\mathrm{V}_{107}$ for PTV & $1.2 \pm 1.1$ & $0.6 \pm 1.3$ & 0.131 \\
\hline Global maximum dose & $56.0 \pm 0.46$ & $55.6 \pm 0.7$ & 0.157 \\
\hline $\mathrm{V}_{30}$ for lung & $29.0 \pm 4.7$ & $30.2 \pm 3.9$ & 0.473 \\
\hline Mean heart dose & $6.6 \pm 0.8$ & $6.4 \pm 1.2$ & 0.725 \\
\hline Monitor units delivered & $775.8 \pm 146.3$ & $758.6 \pm 123.2$ & 0.767 \\
\hline
\end{tabular}




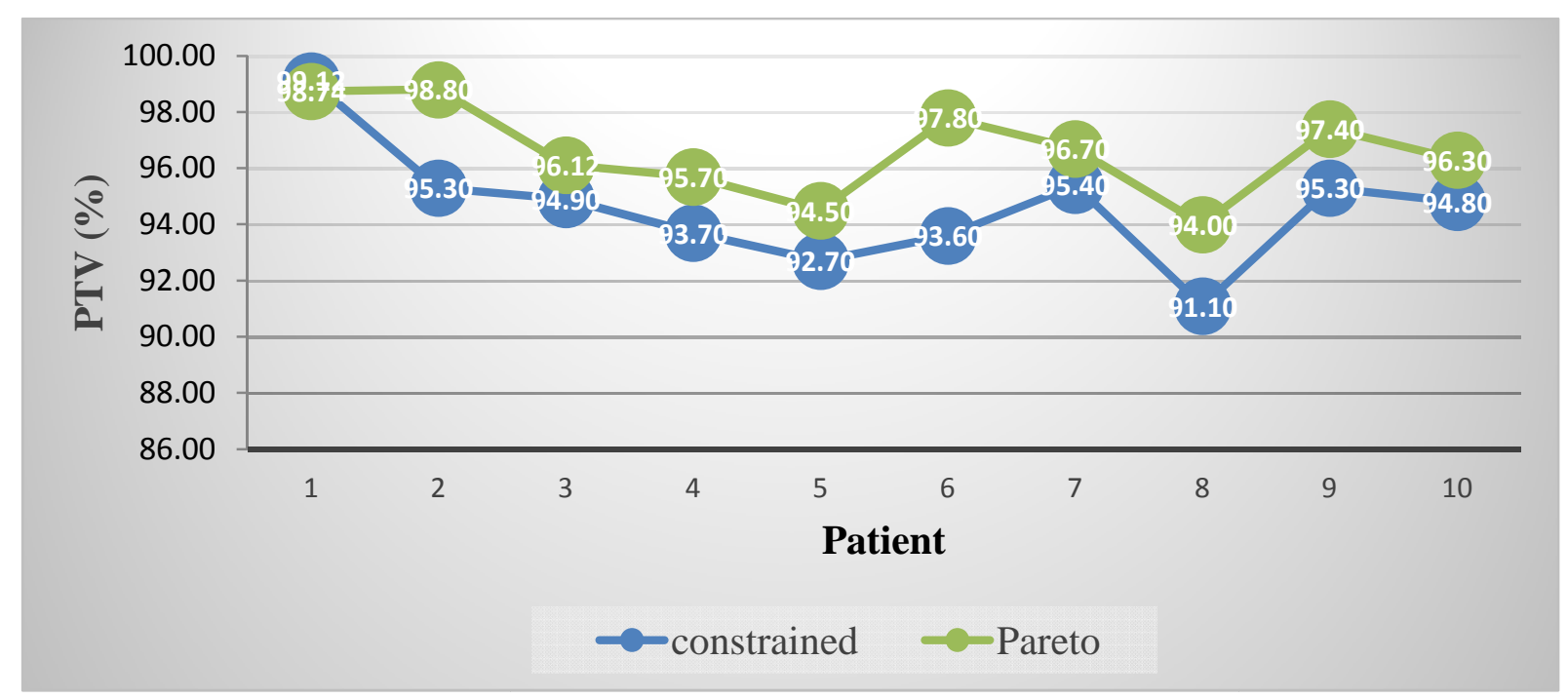

Figure 1. $V_{95}$ for PTV (p-value $\left.\leq \mathbf{0 . 0 0 7}\right)$

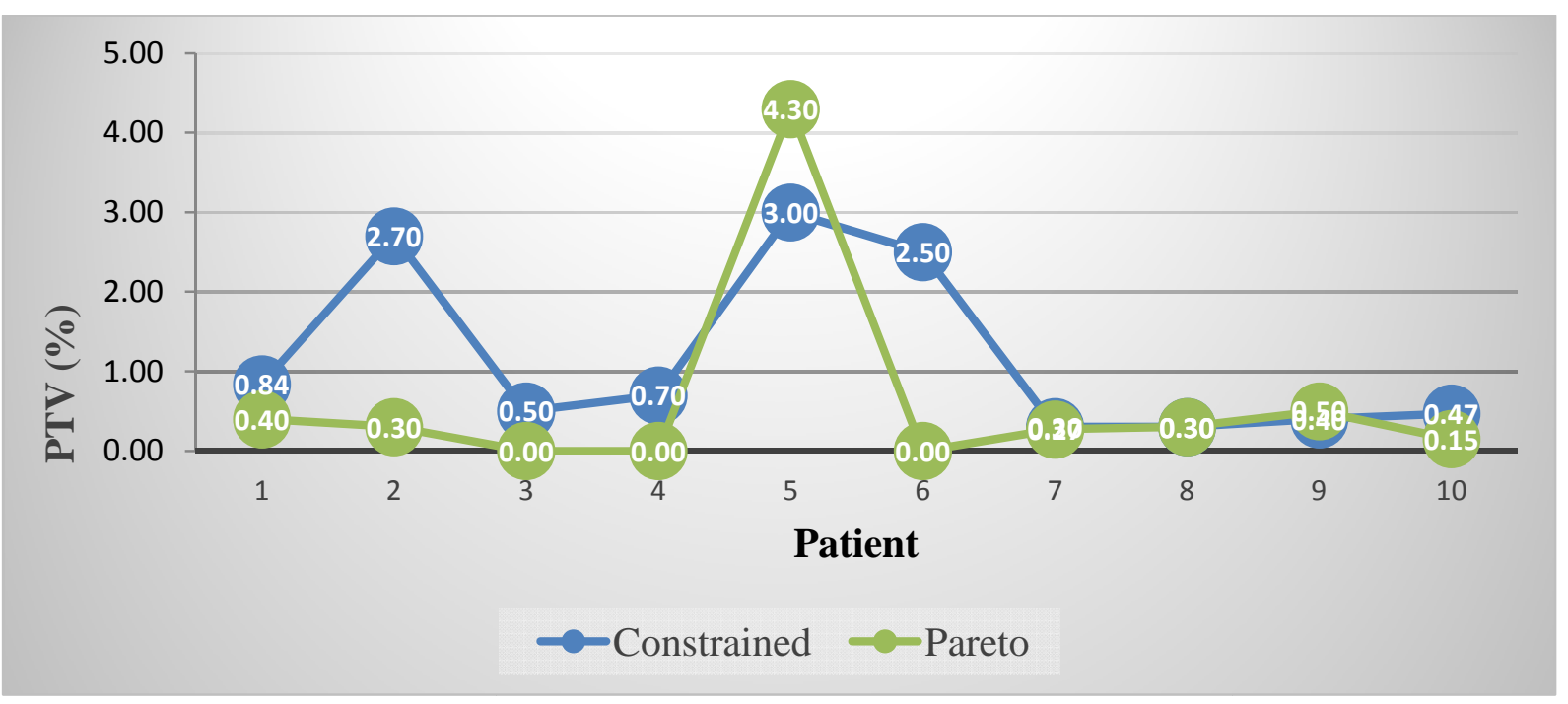

Figure 2. $V_{107}$ for PTV (p-value $\left.\leq \mathbf{0 . 1 3 1}\right)$

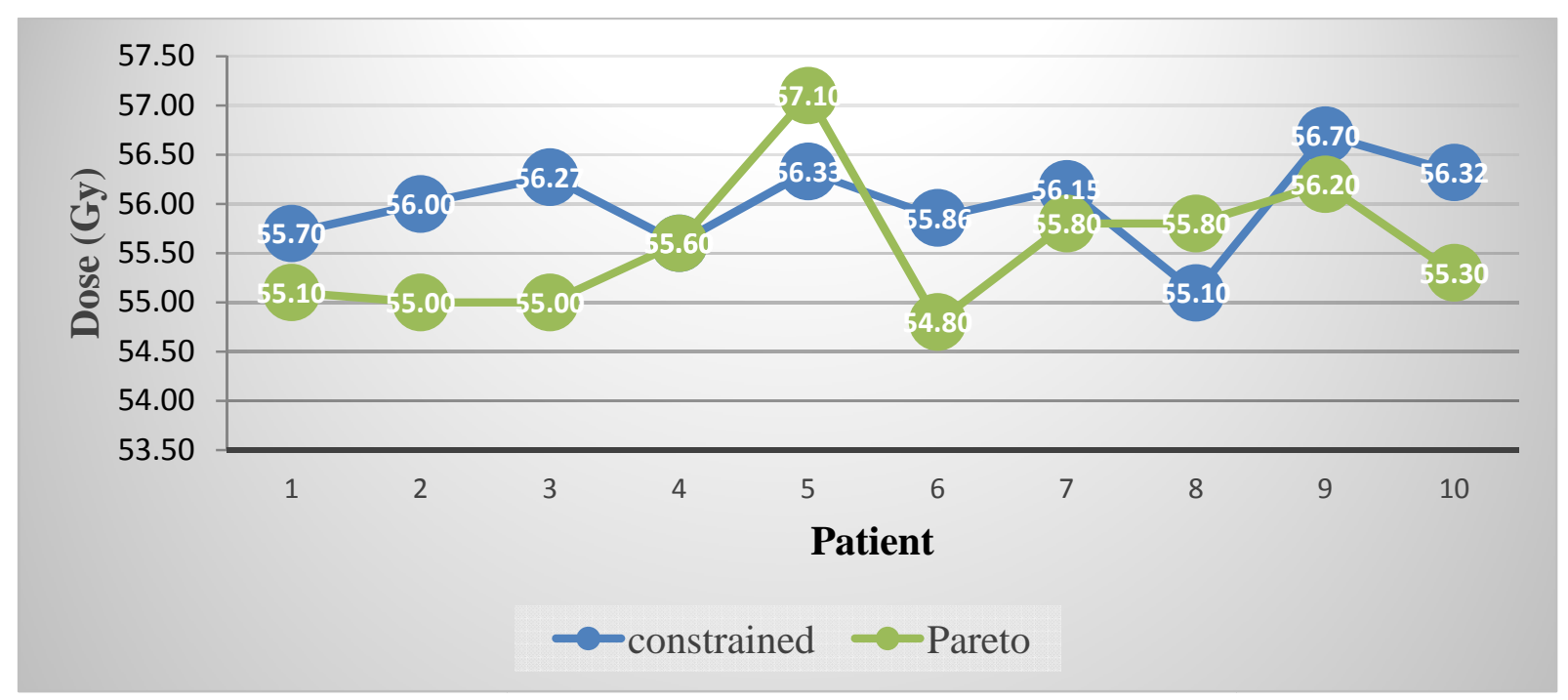

Figure 3. Maximum dose within PTV (p-value $\leq 0.157$ ) 


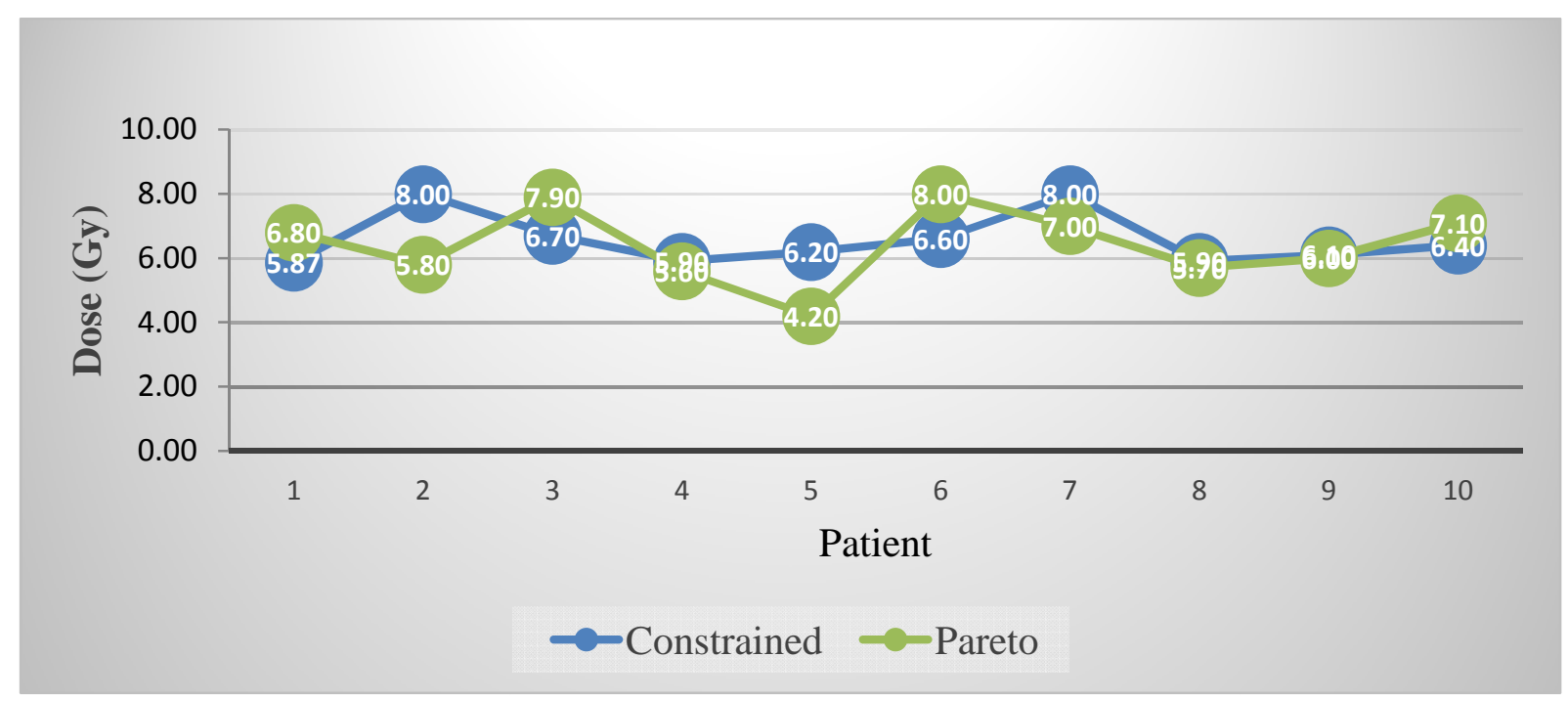

Figure 4. Mean heart dose (p-value $\leq \mathbf{0 . 7 2 5 )}$

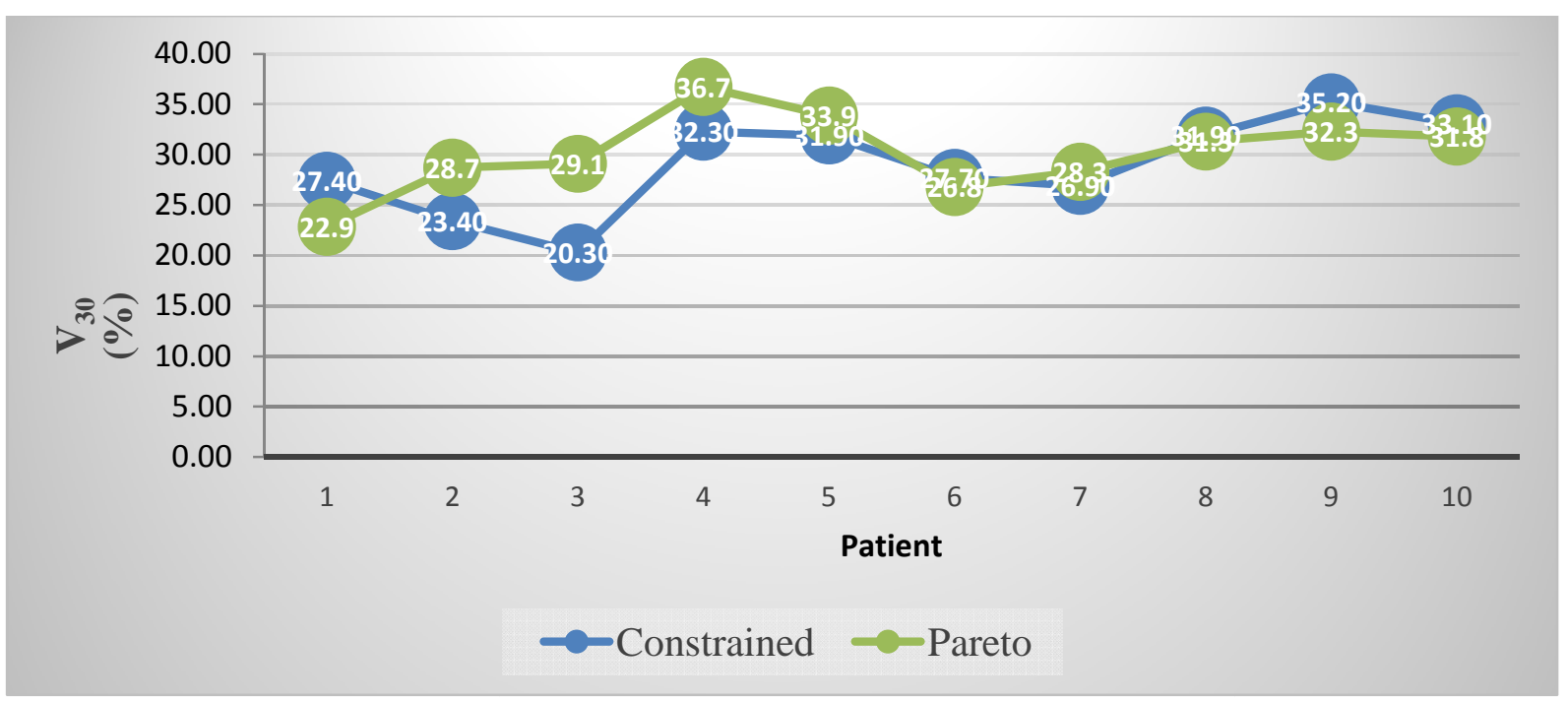

Figure 5. $V_{30}$ for ipsilateral lung (p-value $\leq \mathbf{0 . 4 7 3}$ )

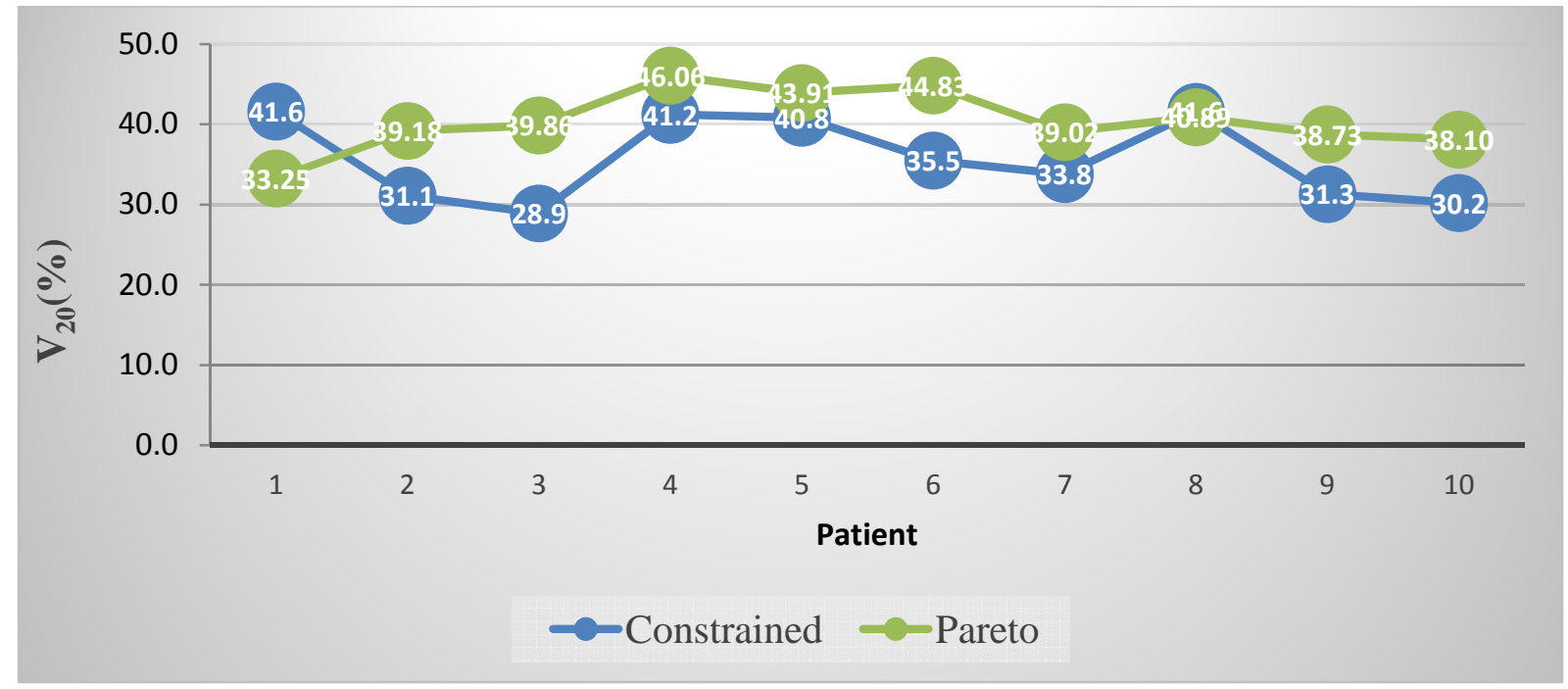

Figure 6. $\mathrm{V}_{20}$ for ipsilateral lung (p-value $\leq \mathbf{0 . 0 6 5 )}$ 


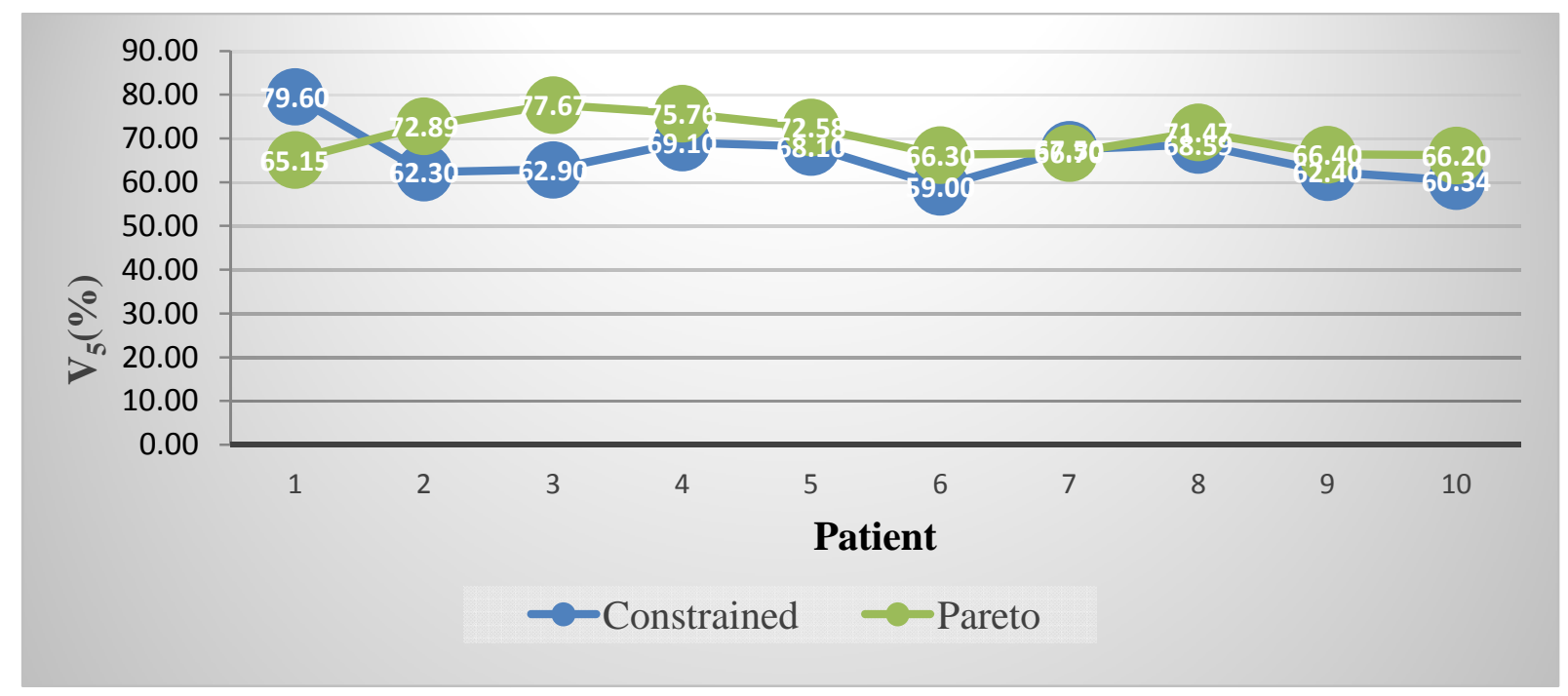

Figure 7. $V_{5}$ for ipsilateral lung (p-value $\left.\leq \mathbf{0 . 0 8 3}\right)$

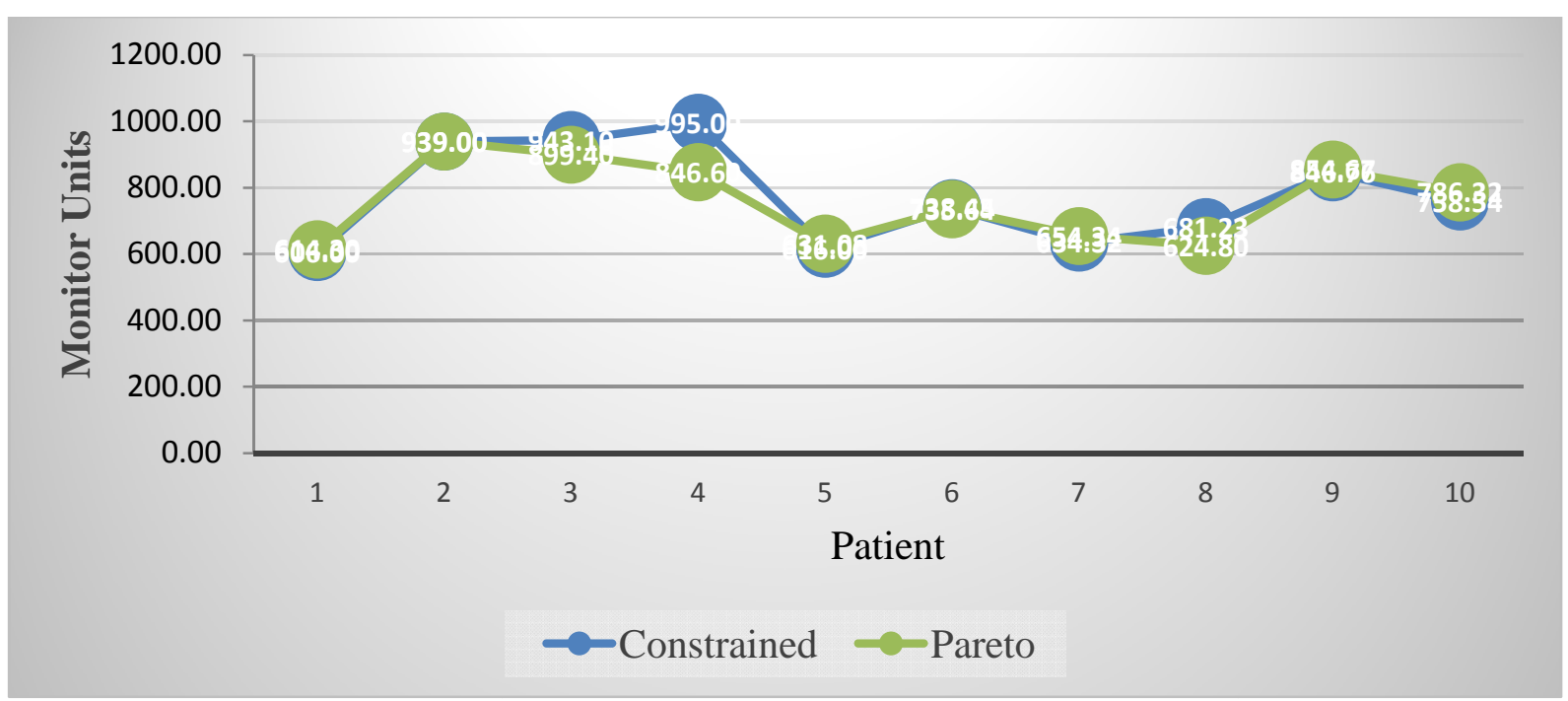

Figure 8. Total monitor units delivered (MU) $(\mathrm{p}$-value $\leq \mathbf{0 . 7 6 7})$

The mean and standard deviation for the various dosimetric quantities are represented in parenthesis. In the constrained mode of optimization, the relative volume of PTV encompassing $95 \%$ of the isodose line ranges from $91.1 \%$ to $99.12 \%(94.59 \pm 2.1)$. For Pareto mode, the range of relative volume of PTV encompassing $95 \%$ of the isodose line is $94.00 \%$ to $98.8 \%(96.61 \pm 1.63)$. The relative volume of PTV encompassed by more than $107 \%$ of prescribed dose for constrained and Pareto mode varied from $0.3 \%$ to $3 \%$ $(1.17 \pm 1.1)$ and from $0.0 \%$ to $4.3 \%(0.62 \pm 1.3)$ respectively. It was found that the global maximum dose for constrained mode varied from 55.1 Gy- 56.7 Gy $(56.00 \pm 0.46)$ and for Pareto mode ranged from 54.8 Gy-57.1 Gy $(55.57 \pm 0.7)$. Mean heart dose for constrained and Pareto mode varied from $5.87 \mathrm{~Gy}$ to $8.00 \mathrm{~Gy}(6.57 \pm 0.81)$ and $4.20 \mathrm{~Gy}$ to $8.00 \mathrm{~Gy}(6.41 \pm 1.17)$ respectively. For constrained and Pareto mode of optimization, mean $\mathrm{V}_{30}, \mathrm{~V}_{20}, \mathrm{~V}_{5}$ values were found to be $29.01,35.6$, and $65.98 \mathrm{~Gy}$ and $30.18,40.38$, and $70.11 \mathrm{~Gy}$ respectively. The total number of monitor units delivered for constrained and
Pareto mode of optimization ranged from 606.0-995.0 $(775.84 \pm 146.34) \quad$ and $\quad 614.3-939 \quad(758.62 \pm 123.15)$ respectively.

\section{Discussion}

There have been various studies either utilising the Pareto tool of optimization or multi-criteria optimization for VMAT. Vadzim et $\mathrm{al}^{11}$ published their study based on biological cost functions for prostate and reported that Monaco VMAT plans reduced median dose to the bladder in VMAT plan. He showed better target conformity when the biological cost functions were used. They said that Pareto represents a convenient optimization tool to explore potential to achieve a desirable balance between doses to target and OARs. Pareto also provides an estimate of the potential reduction of OAR dose and, therefore, may reduce complication probability. They also concluded that the advantage of using biological models in treatment planning is that all the voxels present in the region of 
interest are playing a role in the optimization process instead of the few voxels that receive a disproportionate optimization weight due to point-based constraint violations. Biological cost functions offer more control over the dose distribution than physical cost functions. The statistical evaluations and analysis of the dosimetric outcome of our study are also suggestive of the superiority of Pareto mode especially if we talk about target coverage with comparable dose to OARs. It also suggests that $\mathrm{V}_{95}$ for PTV, $\mathrm{V}_{107}$ for PTV, global maximum dose, mean heart dose, and monitor units delivered improved with their significance depending on p-value for Pareto mode of optimization for left-sided post-mastectomy breast patients. Mean $\mathrm{V}_{95}$ for PTV increased by $2.02 \%$ (2 tailed, pvalue $=0.007)$ which is significant. The mean volume receiving $107 \%$ of the prescribed dose is found to be $0.55 \%$ less for the Pareto mode of optimization ( $p$-value $=0.131)$. The mean dose to heart decreased by $0.16 \mathrm{~Gy}$ ( $\mathrm{p}$-value $=0.725)$; however, the dose to lung increased but not significant except V5 for lung ( $\mathrm{p}$-value $=0.083$ ) which is in line with the concept that low dose local volumes increases in VMAT. As far as monitor units are concerned it decreased for the Pareto mode of optimization. X. Sharon Qi, et $\mathrm{al}^{12}$ in their study have claimed that they were first to use Monaco VMAT for whole-breast planning. They compared VMAT and tomotherapy plans and found that both showed increased conformality and greater sparing of the heart for patients with and without nodal treatment. They reported that a potential problem associated with Monaco VMAT is that it may result in larger dose inhomogeneity when biological model-based optimization is used. They also concluded that the regions of high dose were modest and unlikely to be of any clinical significance. Insensitivity of the biological function to the cold spots leads to cold spots within the breast to maintain the same equivalent uniform dose. However, they have observed that this problem is more prominent for larger targets. In our study, we did not notice any significant inhomogeneity and cold spot in the target volume. Chou-Hsien Lee et $\mathrm{al}^{13}$ in their study evaluated the feasibility and benefit of generalized equivalent uniform doseassisted optimization in volumetric-modulated arc therapy for recurrent head and neck cancer and discussed various limitations like the issue of priority/weight during the optimization process. They advocated that non-utilizing the biological evaluation tools such as TCP/NTCP in spite of potential benefits, may result in inhomogeneity. Vladimir A. Semenenko, et al, ${ }^{14}$ also evaluated the impact of biological optimization in the Monaco TPS on plan quality. They compared the outcome from the Monaco plan with conventional TPS from the same vendor employing dose-based optimization. They demonstrated the utility of various radiobiological dose plan parameters in the Monaco TPS system (Table 1). Our results confirm fact that VMAT may be a better option for tumour topology of the left post-mastectomy breast especially.

\section{Conclusion}

Results of the study show some advantages of Pareto mode optimization over constrained mode for target coverage with comparable OAR doses. Pareto mode has also demonstrated better outcomes in the reduction of high dose volume, reduction of mean heart dose, and reduction in global maximum dose. Pareto mode of optimization may be a method of choice especially for left post-operated breast cancer patients.

\section{References}

1. Bogue J, Wan J, Lavey RS, Parsai EI. Dosimetric comparison of VMAT with integrated skin flash to 3D field-in-field tangents for left breast irradiation. J Appl Clin Med Phys. 2019;20(2):24-29. https://doi.org/10.1002/acm2.12527

2. Dumane VA, Lo YC, Green S. Combination of volumetric-modulated arc therapy (VMAT) and partially wide tangents (PWT) for improved organ sparing in a left-sided and right-sided breast cancer case receiving regional nodal irradiation (RNI): A technical note. Appl Rad Oncol. 2018;7(2):31-38.

3. Byrne M, Archibald-Heeren B, Hu Y, et al. Comparison of semi-automated tangential VMAT with 3DCRT for breast or chest wall and regional nodes. J Appl Clin Med Phys. 2018;19(5):684-693. https://doi.org/10.1002/acm2.12442

4. Sudha SP, Seenisamy R, Bharadhwaj K. Comparison of dosimetric parameters of volumetric modulated arc therapy and three dimensional conformal radiotherapy in post mastectomy patients with carcinoma breast. J Can Res Ther. 2018;14(5):1005-1009. https://doi.org/10.4103/0973-1482.189400

5. Fogliata A, Seppäla J, Reggiori G, et al. Dosimetric trade-offs in breast treatment with VMAT technique. Br J Radiol. 2016;90:1070. https://doi.org/10.1259/bjr.20160701

6. Clements M, Schupp N, Tattersall M, et al. Monaco treatment planning system tools and optimization processes. Medical Dosimetry. 2018;43(2):106-117. https://doi.org/10.1016/j.meddos.2018.02.005

7. Craft D. Multi-criteria optimization methods in radiation therapy planning: a review of technologies and directions. arXiv:1305.1546v1 
8. Craft D, Bortfeld T. How many plans are needed in an IMRT multi- objective plan database? Phys Med Biol. 2008;53(11):27852796. https://doi.org/10.1088/0031-9155/53/11/002

9. Craft D, McQuaid D, Wala J, et al. Multicriteria VMAT optimization. Med Phys. 2012;39(2):686-696. https://doi.org/10.1118/1.3675601

10. RTOG Oncology Group. Breast Cancer Atlas for Radiation Therapy Planning: Consensus Definitions. https://www.nrgoncology.org/Portals/0/Scientific\%20Program/CIRO/Atlases/BreastCancerAtlas_corr.pdf?ver=2018-04-18-144201270

11. Pyshniak V, Fotina I, Zverava A,. Efficiency of biological versus physical optimization for single-arc VMAT for prostate and head and neck cases. J Appl Clin Med Phys. 2014;15(4):39-53. https://doi.org/10.1120/jacmp.v15i4.4514

12. Qi XS, Liu TX, Liu AK, et al. Left-sided breast cancer irradiation using rotational and fixed- field radiotherapy. Med Dosim. 2014;(39):227-234. https://doi.org/10.1016/j.meddos.2014.02.005

13. Lee $\mathrm{CH}$, Jhang ZR, Yeh SA, et al. A planning study for evaluation of feasibility and benefit of generalized equivalent uniform doseassisted optimization in volumetric-modulated arc therapy for recurrent head and neck cancer. Ther Radiol Oncol. 2018;2:36. https://doi.org/10.21037/tro.2018.08.01

14. Semenenko VA, Reitz B, Day E, et al. Evaluation of a commercial biologically based IMRT treatment planning system. Med Phys. 2008;35(12):5851-5860. https://doi.org/10.1118/1.3013556 